\title{
Are we really mapping psychosis risk? Neuroanatomical signature of affective disorders in subjects at ultra high risk
}

\author{
G. Modinos ${ }^{1}$, P. Allen ${ }^{1}$, M. Frascarelli ${ }^{1}$, S. Tognin ${ }^{1}$, L. Valmaggia ${ }^{1}$, L. Xenaki ${ }^{1}$, P. Keedwell ${ }^{2}$, \\ M. Broome ${ }^{3}$, I. Valli ${ }^{1}$, J. Woolley ${ }^{1}$, J. M. Stone ${ }^{1}$, A. Mechelli ${ }^{1}$, M. L. Phillips ${ }^{4}$, P. McGuire ${ }^{1}$ \\ and P. Fusar-Poli ${ }^{1 *}$ \\ ${ }^{1}$ Department of Psychosis Studies, Institute of Psychiatry, King's College London, London, UK \\ ${ }^{2}$ Institute of Psychological Medicine and Clinical Neurosciences, MRC Centre for Neuropsychiatric Genetics and Genomics, Cardiff University, \\ Cardiff, UK \\ ${ }^{3}$ Department of Psychiatry, University of Oxford, Oxford, UK \\ ${ }^{4}$ Department of Psychiatry, Western Psychiatric Institute and Clinic, University of Pittsburgh, Pittsburgh, PA, USA
}

Background. The majority of people at ultra high risk (UHR) of psychosis also present with co-morbid affective disorders such as depression or anxiety. The neuroanatomical and clinical impact of UHR co-morbidity is unknown.

Method. We investigated group differences in grey matter volume using baseline magnetic resonance images from 121 participants in four groups: UHR with depressive or anxiety co-morbidity; UHR alone; major depressive disorder; and healthy controls. The impact of grey matter volume on baseline and longitudinal clinical/functional data was assessed with regression analyses.

Results. The UHR-co-morbidity group had lower grey matter volume in the anterior cingulate cortex than the UHR-alone group, with an intermediate effect between controls and patients with major depressive disorder. In the UHR-co-morbidity group, baseline anterior cingulate volume was negatively correlated with baseline suicidality/ self-harm and obsessive-compulsive disorder symptoms.

Conclusions. Co-morbid depression and anxiety disorders contributed distinctive grey matter volume reductions of the anterior cingulate cortex in people at UHR of psychosis. These volumetric deficits were correlated with baseline measures of depression and anxiety, suggesting that co-morbid depressive and anxiety diagnoses should be carefully considered in future clinical and imaging studies of the psychosis high-risk state.

Received 7 November 2013; Revised 13 March 2014; Accepted 14 March 2014

Key words: Anterior cingulate cortex, co-morbidity, grey matter volume, high risk, neuroimaging, schizophrenia.

\section{Introduction}

Affective disorders are prevalent concomitants of the ultra high risk (UHR) of psychosis (Fusar-Poli et al. 2013a). Symptoms of depression and anxiety are often the primary presenting complaint of the UHR patient rather than the attenuated psychotic symptoms, with a significant impact on psychosocial and emotional functioning (Fusar-Poli et al. 2013a). A recent study in about 500 UHR individuals reported a $73 \%$ prevalence of baseline co-morbid Axis I diagnoses, mostly depressive disorders, in addition to the attenuated psychotic symptoms (Fusar-Poli et al. 2014b). Depressive and anxiety symptoms may chronologically

\footnotetext{
* Address for correspondence: P. Fusar-Poli, M.D., Ph.D., Department of Psychosis Studies, Institute of Psychiatry, Box PO 63, 16 De Crespigny Park, London SE5 8AF, UK.

(Email: paolo.fusar-poli@kcl.ac.uk)
}

predate the onset of attenuated psychotic symptoms (Fusar-Poli et al. 2013a), reflecting core psychopathological features during the prodromal phases of psychosis (Mishara \& Fusar-Poli, 2013). Such symptoms pose challenges in the clinical management of those who attend high-risk services (Fusar-Poli et al. 2013a), and make a negative impact on the longitudinal prognosis of those who meet criteria for UHR for psychosis (Demjaha et al. 2012; Salokangas et al. 2012). However, the contribution of affective symptoms to UHR neurobiology remains unknown.

Depressive and anxiety disorders have specific neuroanatomical, neurofunctional and neurochemical features (Radua et al. 2010; Sacher et al. 2012); therefore the high prevalence of these symptoms in UHR cohorts could potentially confound previous neurobiological findings in these subjects. Consequently, this heterogeneity across individual neuroimaging studies (FusarPoli et al. 2011, 2012) may prevent the identification of 
reliable markers of psychosis risk. The lack of specificity regarding the neurobiological correlates of UHR states (Fusar-Poli et al. 2014c) may ultimately affect the validity of the paradigm itself and prevent further developments in the field. A recent consensus paper indicated that the lack of studies testing the validity of the UHR paradigm against other psychiatric samples (such as those affected by depressive disorders) prevented its final inclusion in the recent Diagnostic and Statistical Manual of Mental Disorders, fifth edition (DSM-5) (Yung et al. 2012). Furthermore, criterion $\mathrm{E}$ of the proposed 'attenuated psychosis syndrome' requires that symptoms are not better explained by any other DSM diagnosis (Tsuang et al. 2013). Consequently, the question of the clinical and neuroanatomical specificity of UHR symptoms as opposed to depressive or anxiety symptoms is of crucial nosographic relevance (van Os \& Murray, 2013).

To date, imaging studies have assumed that UHR states are characterized by common neuroanatomical alterations. However, this seems unlikely given the wide range of symptomatic co-morbidities that are present in UHR cohorts and the heterogeneity of clinical outcomes. Human in vivo neuroimaging studies have shown that UHR states and affective disorders such as major depressive disorder (MDD) each have distinct deleterious effects on the brain and associated cognitive and social behaviour (Fusar-Poli et al. 2011; Bora et al. 2012). In MDD, imaging studies report grey matter volume (GMV) reductions most robustly in the rostral anterior cingulate cortex (ACC) (Drevets, 2000, 2001; Bora et al. 2012; Sacher et al. 2012). In UHR cohorts, quantitative brain imaging studies report substantial GMV deficits compared with healthy controls, most consistently found in the temporal and frontal cortices, the limbic system and the cerebellum (Pantelis et al. 2003; Borgwardt et al. 2007; Meisenzahl et al. 2008). To our knowledge, the neuroanatomical impact of these UHR co-morbidities has never been studied.

Here we tested the hypothesis that co-morbid depressive and anxiety disorders make an impact upon the neuroanatomy of psychosis risk. In the light of the high co-morbid prevalence of these disorders, we examined their contribution to grey-matter UHR abnormalities using a whole-brain voxel-based morphometry method (VBM). We included 121 men and women from four groups: (1) UHR alone; (2) UHR with anxiety or depressive co-morbidity; (3) healthy controls (HC); and (4) MDD alone. The latter group was selected to test the validity (Tsuang et al. 2013) and specificity (Yung et al. 2012) of UHR neuroanatomy in comparison with other psychiatric diagnoses. Our first hypothesis was that the UHR group as a whole would show GMV decreases relative to HCs in line with previous findings (Fusar-Poli et al. 2011), but that stratifying the sample on the basis of comorbidity would reveal different patterns of GMV change, particularly in the ACC (Bora et al. 2012). Given the higher prevalence of attenuated depressive symptoms in the UHR group compared with HCs (Fusar-Poli et al. 2014b), we specifically hypothesized that the UHR co-morbidity group would show an intermediate pattern of ACC grey-matter change between MDD and UHR-alone patients. Second, we hypothesized that neuroanatomical alterations in UHR subjects with co-morbidity would be correlated with baseline and longitudinal clinical and functional measures (Borgwardt et al. 2008; Fornito et al. 2008).

\section{Method}

Sample

UHR group

Individuals meeting Personal Assessment and Crisis Evaluation (Yung et al. 1998) criteria for an at-risk mental state for psychosis (UHR) were recruited from a prodromal clinical service, the Outreach and Support in South London service (OASIS) (Fusar-Poli et al. 2013b). Details of these criteria are appended in the online Supplementary Method.

The total sample consisted of 52 UHR subjects ( mean age $=25.3$ years, s.D. $=4.3$ years, 62 $\%$ males), whose individual structural magnetic resonance imaging (MRI) scans were collected at baseline from consecutive referrals to OASIS (Broome et al. 2005). All UHR participants were antipsychotic naive at the time of scanning. The UHR group was divided according to depression and anxiety co-morbidity ('UHR co-morbidity': $n=32$, mean age $=23.6$ years, S.D. $=$ 4.2 years, 69\% males; 'UHR alone': $n=20$, mean age $=$ 23.3 years, S.D. $=4.8$ years, $60 \%$ males), in line with our previous behavioural multicentre study (FusarPoli et al. 2014b). Current DSM diagnoses of Axis I co-morbidity (anxiety, depression, and anxiety plus depression disorders) were assessed during the initial assessment at the OASIS clinic using the Structured Clinical Interview for DSM-IV (SCID) (First et al. 1996). All UHR subjects were followed up clinically (for at least 2 years). Clinical (transition to psychosis) and functional (good/poor) outcomes were determined with the Comprehensive Assessment of At Risk Mental States (CAARMS; Yung et al. 2005) and Global Assessment of Functioning (GAF) (APA, 1994), respectively, while the sociodemographic characteristics were recorded through unstandardized and locally developed questionnaires (Schennach-Wolff et al. 2009). We accessed information on the treatments that had been offered to UHR subjects during their clinical 
management at the OASIS clinic (i.e. from baseline to follow-up) from the OASIS patient records. Therefore, the clinical assessment was carried out at two time points (baseline and follow-up), and the MRI was only carried out at baseline.

\section{MDD group}

A total of 23 individuals with MDD (mean age $=44.6$ years, s.D. $=5.5$ years, $13 \%$ males) were recruited from services at the South London and Maudsley National Health Service Trust. MDD was assessed with the SCID (First et al. 1996), and diagnosed using DSM-IV criteria (APA, 1994), by the psychiatrist responsible for the patients' management at the Maudsley Hospital, London. Ethical approval was obtained from the Ethical Committee of the South London and Maudsley Trust and Institute of Psychiatry. Written consent was obtained from all subjects prior to participation in the study. Exclusion criteria included a history of head injury, illicit substance abuse, and a score of less than 24 on the Mini-Mental State Examination (Folstein et al. 1975).

HC group

A total of 46 healthy volunteers (mean age $=25.3$ years, S.D. $=4.3$ years, $69 \%$ males) were recruited via advertisements in the local media. All individuals lived in the same borough of London as the clinical participants (Lambeth/Southwark). The HC sample was comparable with the total UHR group for gender and age.

For all participants, exclusion criteria included other past/present diagnosis of Axis I psychiatric illnesses, past/present/familiar history of neurological illness, intellectual impairments, medical illness, alcohol or other substance abuse or dependence (defined using DSM-IV criteria), and pregnancy.

\section{MRI acquisition}

MR images were acquired using a GE Signa 1.5 T MR system (General Electric, USA) at the Maudsley Hospital, London. Subjects were scanned with a T1weighted three-dimensional spoiled gradient recall sequence with $1.5 \mathrm{~mm}$ thickness and 0 gap (repetition time $=3000 \mathrm{~ms}$, inversion time $=300 \mathrm{~ms}$, echo time $=$ $40 \mathrm{~ms}$ ), producing 124 slices.

\section{Data analysis}

Sociodemographic, clinical and functional data

Differences in demographic, clinical and functional characteristics between groups at baseline and followup cross-sectionally were examined using one-way analysis of variance for parametric data and a $\chi^{2}$ test for non-parametric data. The interaction between CAARMS assessment time (baseline, follow-up) and group (UHR alone, UHR co-morbidity) was tested with a repeated-measures analysis of variance. All analyses were performed using SPSS version 20.0 for Mac (IBM, USA).

Image preprocessing

Structural images were preprocessed using VBM implemented in the SPM8 software package (Wellcome Department of Cognitive Neurology, UK) running under Matlab 8.1 (The Mathworks, USA). Further details on image pre-processing are appended in the Supplementary Method.

\section{Image analysis}

To test our first hypothesis, an analysis of variance was designed using SPM8 (http://www.fil.ion.ucl.ac.uk/ spm/software/spm8/) to compare the grey matter images from participants with UHR co-morbidity, MDD alone and UHR alone and HC participants. We modelled age and gender as covariates of no interest to identify regionally specific differences that were not confounded by these variables. The proportional scaling option was used to identify regional changes that were not confounded by global differences in GMV. Statistical inferences were made at $p<0.05$ family-wise error (FWE) across the whole brain, with cluster-level correction as determined by the Monte Carlo simulation program implemented in AlphaSim (Song et al. 2011) (http://afni.nimh.nih.gov/pub/dist/ doc/manual/AlphaSim.pdf) $(p=0.001, \mathrm{k}=123)$. For the comparison of UHR alone and UHR co-morbidity, based on our a priori hypothesis of an effect in the ACC derived from studies in MDD and in the early phases of psychosis (Borgwardt et al. 2008; Bora et al. 2012; Radua et al. 2012; Sacher et al. 2012), we created a pre-defined anatomical mask including the bilateral ACC using Automated Anatomical Labeling as implemented in the WFU_Pickatlas toolbox (including 2713 voxels). The extent threshold of the cluster for this region-of-interest analysis (ROI) was also determined by Monte Carlo simulation $(p=0.005, \mathrm{k}=27)$ to ensure an FWE correction level of $p<0.05$.

Baseline and longitudinal correlations of imaging data with clinical and functional measures

To test our second prediction, correlations between baseline ACC volume and baseline psychopathology (CAARMS), as well as longitudinal CAARMS scores, were tested using Pearson's product-moment correlation in SPSS (SPSS Inc., USA). 
In addition to the standard diagnostic subscales of the CAARMS (unusual thought content, perceptual abnormalities, speech disorders), we a priori focused our correlational analyses on the avolition, disorganized behaviour, depression, suicidality/self-harm, anxiety and obsessive-compulsive disorder (OCD) subscales of the CAARMS, since we previously showed that co-morbid depressive and anxiety disorders had a significant impact on these (Fusar-Poli et al. 2014b). For completeness, we also analysed the other psychopathological CAARMS domains, correcting for multiple comparisons. Outliers were identified with visual inspections of regression lines and the use of Cook's distance test. Predictions of transition and functional outcomes (poor/good) of UHR individuals based on ACC volume were conducted by using Cox regression analyses and hazard ratios were estimated. CAARMS subscales were treated as continuous variables consistent with prior research (e.g. Yung et al. 2005; Thompson et al. 2011; Valmaggia et al. 2013). The poor and good functional outcome groups were defined by a median split of scores, in line with previous studies in schizophrenia (Ho et al. 2003; Spellmann et al. 2012; Wulff et al. 2012), and in UHR individuals (Velthorst et al. 2013).

\section{Results}

\section{Sociodemographic, clinical and functional data}

There were no differences between the UHR-alone, UHR-co-morbidity and HC groups in age or gender. However, the MDD sample was significantly older and had significantly more women $\chi_{6}^{2}=61.443$, $p<0.001)$ than the other three groups $\left(F_{3,120}=120.944\right.$, $p<0.001$; Bonferroni post-hoc correction was $p<0.001$ for MDD $v$. UHR alone, MDD $v$. UHR co-morbidity, and MDD $v$. HC).

The clinical characteristics of the UHR subjects (cross-sectionally and longitudinally) are detailed in Table 1. Of the 52 UHR subjects, 32 (62\%) had an Axis I co-morbid diagnosis of depressive or anxiety disorder in addition to the at-risk signs and symptoms. Specifically, 26 out of $32(81 \%)$ had a co-morbid diagnosis of depression alone or in association with anxiety disorders. Anxiety disorders alone were less frequent, found in six participants out of $32(19 \%)$. UHR participants with and without co-morbidity did not differ on interval between baseline and follow-up assessments. The UHR groups differed at baseline on disorganized speech $\left(F_{1,47}=4.202, p=0.046\right)$, with higher levels in the UHR-co-morbidity group. The UHR-co-morbidity group also had higher levels of avolition $\left(F_{1,44}=5.509\right.$, $p=0.023)$ and depression $\left(F_{1,44}=3.450, p=0.070\right.$, trend level) (Table 1; see online Supplementary Table S1 for the full baseline psychopathological assessment).

At follow-up, nine UHR individuals (17\%) had developed psychosis, while the remaining 43 (83\%) had not. Average follow-up time was $5.5(\mathrm{SD}=2.9)$ years. The UHR-alone and UHR-co-morbidity groups did not differ in the number of transitions, or the proportion of good and poor functional outcomes (GAF median split=67.55). At follow-up, there were no significant symptom differences between the UHR-alone and UHR-co-morbidity groups (Table 1; see online Supplementary Table S1 for the full follow-up psychopathological assessment). However, there was a time $\times$ group interaction, with an association between UHR co-morbidity and larger improvements in depression levels $\left(F_{1,33}=4.699, \quad p=0.037\right)$ over time compared with UHR alone. Of note, there were significant differences in the treatments offered to the two groups during their clinical management at the OASIS clinic (i.e. from baseline to follow-up). The UHR-comorbidity individuals were more likely to be treated with antipsychotics $\left(\chi_{1}^{2}=5.953, p=0.015\right)$ and antidepressants $\left(\chi_{1}^{2}=5.215, p=0.022\right)$, and less likely to be treated with cognitive psychological therapies without adjunctive medications $\left(\chi_{2}^{2}=13.939, p=0.001\right)$ than the UHR-alone group (Table 1 ).

\section{Impact of depressive and anxiety co-morbid disorder on UHR neuroanatomy}

Patients with MDD showed GMV reductions, compared with HC participants with a whole-brain analysis, in a large medial frontal cluster including the orbital gyrus, ACC and medial frontal gyrus, as well as in the middle temporal gyrus and the inferior parietal lobe (online Supplementary Table S2 and Fig. 1a). MDD patients also showed reductions in medial frontal and inferior parietal clusters compared with UHR-co-morbidity individuals (Fig. 1b), and similarly with UHR-alone subjects (Fig. 1c).

There were GMV reductions in UHR-co-morbidity compared with UHR-alone subjects as revealed by a whole-brain analysis in the medial frontal gyrus and the fusiform gyrus (online Supplementary Table S2). The ROI analysis based on an anatomical ACC mask showed a significant reduction in GMV in the UHR-co-morbidity group relative to the UHR-alone group in a cluster comprising the subgenual prefrontal cortex and the pregenual ACC (online Supplementary Table S2 and Fig. 2). Further analysis confirmed a trend across the four groups, with MDD patients showing the most pronounced decreases relative to $\mathrm{HCs}$, followed by the UHR-co-morbidity and UHR-alone groups (main effect of group: $F_{3,120}=15.422, p<0.001$; Bonferroni post-hoc comparisons: $\mathrm{MDD}<\mathrm{HC}, p<0.001$; 
Table 1. Baseline and longitudinal clinical (CAARMS) ${ }^{\mathrm{a}}$, functional (GAF) and outcome profiles of the UHR sample

\begin{tabular}{|c|c|c|c|c|c|}
\hline Characteristic & $\begin{array}{l}\text { UHR total } \\
(n=52)\end{array}$ & $\begin{array}{l}\text { UHR-alone } \\
\text { group }(n=20)\end{array}$ & $\begin{array}{l}\text { UHR-depressive or } \\
\text { anxiety co-morbidity } \\
\text { group }(n=32)\end{array}$ & $F$ or $\chi^{2}$ & $p$ \\
\hline \multicolumn{6}{|l|}{ Baseline } \\
\hline Disorder of thought content & $3.4(1.4)$ & $3.7(1.5)$ & $3.3(1.3)$ & 1.288 & 0.262 \\
\hline Perceptual abnormalities & $2.5(1.9)$ & $2.7(2.1)$ & $2.5(1.8)$ & 0.144 & 0.706 \\
\hline Disorganized speech & $1.5(1.4)$ & $1.0(1.1)$ & $1.8(1.4)$ & 4.202 & 0.046 \\
\hline Avolition & $2.6(1.7)$ & $1.8(1.7)$ & $3.0(1.6)$ & 5.509 & 0.023 \\
\hline Disorganized behaviour & $1.0(1.3)$ & $1.1(1.2)$ & $0.7(1.3)$ & 1.032 & 0.315 \\
\hline Depression & $2.5(1.8)$ & $1.9(1.5)$ & $2.9(1.8)$ & 3.450 & 0.070 \\
\hline Suicidality & $1.8(1.6)$ & $1.9(1.8)$ & $1.7(1.5)$ & 0.104 & 0.749 \\
\hline Anxiety & $3.2(1.6)$ & $3.2(1.6)$ & $3.3(1.6)$ & 0.041 & 0.841 \\
\hline OCD & $1.4(1.5)$ & $1.1(1.3)$ & $1.6(1.5)$ & 1.289 & 0.262 \\
\hline GAF, good & $68.7(8.4)$ & $67.2(7.2)$ & $69.4(9.1)$ & 0.410 & 0.528 \\
\hline GAF, poor & $50.0(6.0)$ & $50.3(7.5)$ & $49.9(5.4)$ & 0.021 & 0.887 \\
\hline \multicolumn{6}{|l|}{ Longitudinal follow-up } \\
\hline Disorder of thought content & $1.7(1.9)$ & $1.7(1.9)$ & $1.8(1.7)$ & 0.017 & 0.898 \\
\hline Perceptual abnormalities & $2.8(1.8)$ & $2.0(1.8)$ & $1.5(1.8)$ & 0.647 & 0.427 \\
\hline Disorganized speech & $1.6(1.4)$ & $1.2(1.6)$ & $1.1(1.4)$ & 0.116 & 0.735 \\
\hline Avolition & $2.6(1.8)$ & $1.0(1.3)$ & $1.3(1.5)$ & 0.271 & 0.606 \\
\hline Disorganized behaviour & $1.3(1.5)$ & $0.1(0.5)$ & $0.3(0.9)$ & 0.192 & 0.664 \\
\hline Depression & $2.8(1.8)$ & $1.2(1.7)$ & $1.5(1.8)$ & 0.370 & 0.547 \\
\hline Suicidality/self-harm & $2.1(1.5)$ & $0.6(1.1)$ & $0.7(1.1)$ & 0.109 & 0.743 \\
\hline Anxiety & $3.4(1.5)$ & $1.1(1.6)$ & $1.4(1.6)$ & 0.141 & 0.710 \\
\hline OCD & $1.7(1.5)$ & $0.6(1.2)$ & $0.7(1.3)$ & 0.053 & 0.820 \\
\hline GAF, good & $80.9(8.4)$ & $79.4(7.8)$ & $81.9(9.1)$ & 0.429 & 0.520 \\
\hline GAF, poor & $52.8(9.0)$ & $47.8(3.8)$ & $54.6(9.7)$ & 2.288 & 0.149 \\
\hline \multicolumn{6}{|l|}{ Focused interventions, $n(\%)^{\mathrm{b}}$} \\
\hline CBT only & $17(37.0)$ & 12 (66.7) & $5(17.9)$ & & \\
\hline Combination $^{c}$ & $28(60.9)$ & $5(27.8)$ & $23(82.1)$ & 13.939 & 0.001 \\
\hline Monitoring only & $1(2.2)$ & $1(5.6)$ & 0 & & \\
\hline \multicolumn{6}{|l|}{ Antidepressants, $n(\%)^{\mathrm{b}, \mathrm{d}}$} \\
\hline Yes & $14(30.4)$ & 2 (11.1) & $12(42.9)$ & 5.215 & 0.022 \\
\hline No & $32(69.6)$ & $16(88.9)$ & $16(57.1)$ & & \\
\hline \multicolumn{6}{|l|}{ Antipsychotics, $n(\%)^{\mathrm{e}, \mathrm{f}}$} \\
\hline Yes & $21(44.7)$ & $4(22.2)$ & $17(58.6)$ & 5.953 & 0.015 \\
\hline No & $26(55.3)$ & $14(77.8)$ & $12(41.4)$ & & \\
\hline Follow-up time, years & $5.5(2.9)$ & $5.8(2.5)$ & $5.3(3.1)$ & 0.414 & 0.523 \\
\hline Transitions, $n(\%)$ & 9 (17.3) & $3(15.0)$ & $6(18.8)$ & 0.014 & 0.617 \\
\hline
\end{tabular}

CAARMS, Comprehensive Assessment of the At-Risk Mental State; GAF, Global Assessment of Functioning; UHR, ultra high risk of psychosis; OCD, obsessive-compulsive disorder; CBT, cognitive-behavioural therapy; OASIS, Outreach and Support in South London service.

Data are given as mean (standard deviation) unless otherwise indicated.

${ }^{\text {a }}$ For the whole CAARMS subscales, see online Supplementary Tables S2 and S3.

${ }^{\mathrm{b}}$ Reliable information on focused interventions and antidepressant medication was missing from six UHR participants (two UHR alone+four UHR co-morbidity).

${ }^{\mathrm{c}}$ Combination of CBT and medication (antidepressants or antipsychotics).

d Antidepressants: exposure to any antidepressant at any point from OASIS intake until discharge from the service.

${ }^{\mathrm{e}}$ Reliable information on antipsychotic medication was missing from five UHR participants (two UHR alone+three UHR co-morbidity).

${ }^{\mathrm{f}}$ Antipsychotics: exposure to any antipsychotic at any point from OASIS intake until discharge from the service. 


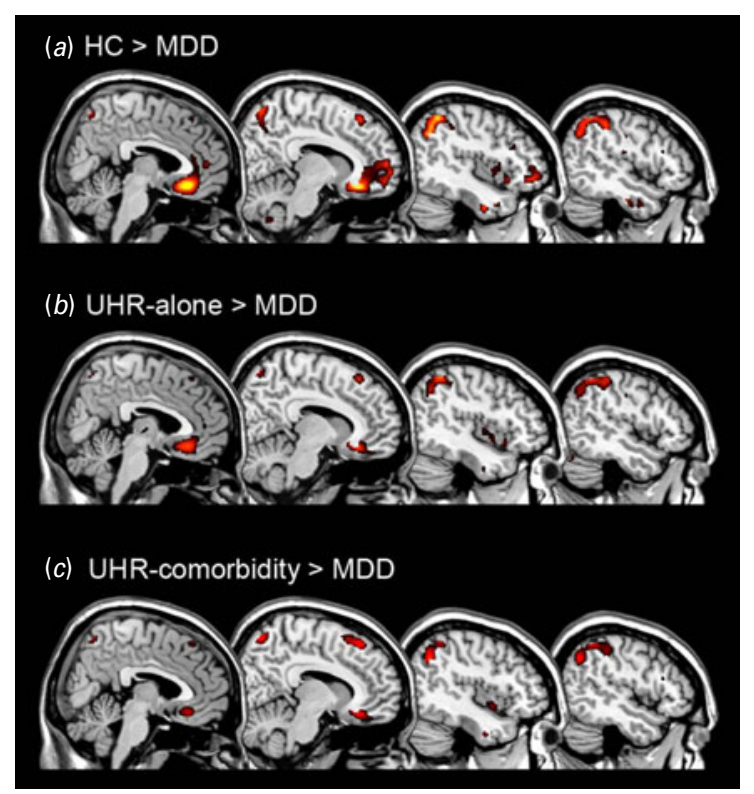

Fig. 1. Group differences in grey matter volume. Differences between healthy control (HC) participants and major depressive disorder (MDD) patients $(a)$, between patients at ultra high risk (UHR) of psychosis alone and MDD patients (b), and between UHR patients with depressive or anxiety co-morbidity and MDD patients $(c)(p<0.05$ after family-wise error correction).

MDD $<$ UHR co-morbidity, $p<0.001 ; \quad \mathrm{MDD}<\mathrm{UHR}$ alone, $p<0.001$; UHR co-morbidity $<\mathrm{HC}, \quad p=0.027$; UHR co-morbidity $<$ UHR alone, $p=0.046$; and $\mathrm{HC}<$ UHR alone, $p=0.827)$. We found no effect of age $\left(F_{1,120}=1.355, p=0.247\right)$ or gender $\left(F_{1,120}<1\right.$, N.s. $)$ in the analyses.

The results of the comparisons between UHR (combined) versus $\mathrm{HC}$, UHR alone versus $\mathrm{HC}$ and UHR co-morbidity versus HC are reported in the online Supplementary Results, and displayed in online Supplementary Fig. S1 $a-c$.

\section{Baseline and longitudinal correlations of ACC volume with clinical and functional measures}

In UHR-co-morbidity participants, and using a priori CAARMS subscales on the basis of previous metaanalytical results (Fusar-Poli et al. 2014b), ACC volume was negatively correlated with baseline suicidality/ self-harm $(r=-0.454, p=0.013)$ and OCD symptoms $(r=-0.386, p=0.039)$. Cook's D test was applied to rule out the influence of potential outliers. This correction strengthened the significance of the correlations in both cases (suicidality/self-harm: $r=-0.494, p=0.009$; OCD symptoms: $r=-0.628, p<0.001$ ) (Fig. 3). There were no correlations at baseline or follow-up for either the UHR-alone group or the UHR groups combined. ACC volume did not predict clinical transition or functional outcomes in either UHR subgroup (see online Supplementary Table S3). However, as noted above, this group was receiving more intensive treatments than the UHR-alone group over the duration of the study.

For completeness, we tested in a collateral analysis whether the above findings survived after removing UHR subjects with anxiety alone from the UHRco-morbidity group. Full results are presented in the online Supplementary Results.

\section{Discussion}

To our knowledge, this is the first study to examine the impact of depressive and anxiety co-morbidity on the neuroanatomy of subjects at UHR of psychosis.

Based on previous MRI studies of UHR samples (Wood et al. 2008; Fusar-Poli et al. 2011; Mechelli et al. 2011), our first hypothesis was that the UHR group as a whole would show GMV decreases relative to HCs, but that stratifying the sample on the basis of co-morbidity would reveal different patterns of GMV change. In line with a recent meta-analysis (Fusar-Poli et al. 2011), UHR individuals as a whole showed decreased GMV in frontal regions, including the rolandic operculum, inferior frontal gyrus, medial frontal gyrus and orbitofrontal gyrus. However, when they were clinically stratified according to the presence or absence of depressive and anxiety comorbidity, the co-morbid group showed further reductions in a temporal region. Furthermore, the direct comparison between UHR-co-morbidity and UHR-alone participants at the whole-brain level showed a significant reduction in UHR-co-morbidity participants in the medial frontal gyrus and the fusiform gyrus. In addition, the ROI analysis on this comparison showed a significant reduction in UHR individuals with co-morbid depressive and anxiety disorders in the hypothesized ACC region. As hypothesized, UHR-co-morbidity individuals showed an intermediate effect between patients with MDD alone (who showed the most pronounced decreases in this region) and UHR alone, suggesting that GMV decreases in the ACC are associated with an increased severity of depressive symptomatology. Future studies incorporating measures of severity of depressive symptoms in an MDD group may help expand this finding.

The ACC region implicated in our study comprised the subgenual prefrontal cortex and pregenual ACC. Prior evidence on the neuroanatomical basis of depressive symptomatology has typically highlighted ACC abnormalities as one of the most robust characteristics of the pathophysiology of MDD (Drevets, 2001; Bora et al. 2012; Sacher et al. 2012). Grey matter abnormalities in the ACC region have also been reported in 


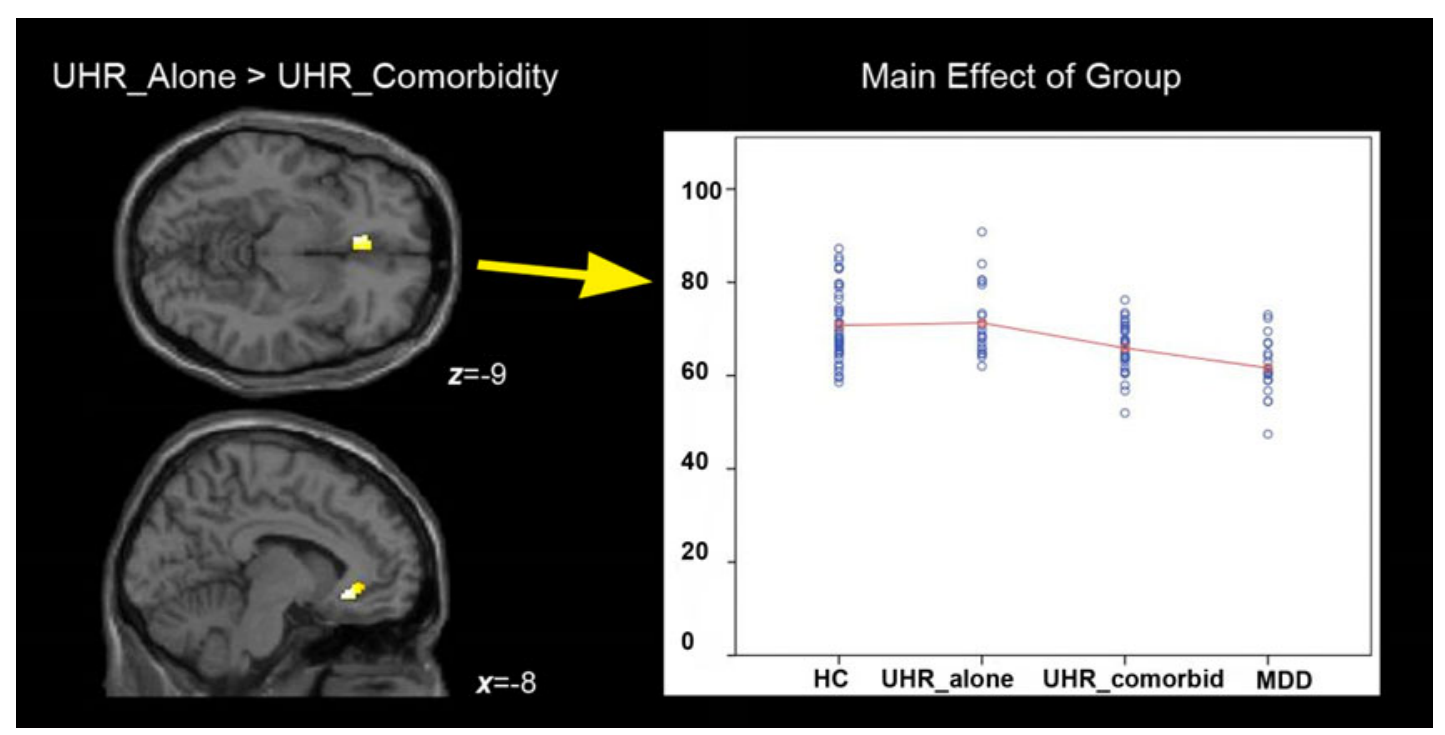

Fig. 2. Effect of co-morbid depression and anxiety on anterior cingulate volume. Differences between ultra-high-risk (UHR) individuals with (UHR co-morbidity) and without (UHR alone) depressive or anxiety co-morbidity, with a region-of-interest approach on the anterior cingulate gyrus ( $p<0.05$ after family-wise error correction). UHR-co-morbidity had less grey matter volume in the anterior cingulate cortex [MNI (Montreal Neurological Institute) coordinates x, y and z: $-8,27$ and -9 , respectively]. For visualization purposes, effects are displayed at $p<0.05$ uncorrected. The plot shows mean grey matter volumes for the four groups derived from the main effect of group results; values on the $\mathrm{y}$-axis refer to $\mathrm{mm}^{3}$ per voxel. HC, Healthy control; MDD, major depressive disorder.

UHR samples regardless of their co-morbidity status (Borgwardt et al. 2008; Fornito et al. 2008; Fusar-Poli et al. 2011; Radua et al. 2012). The detection of abnormalities in this region, which were more severe in the UHR-co-morbidity group, suggests a dysfunction within the limbic portions of the ACC that are known to modulate subcortical limbic and brainstem structures that mediate emotional and social behaviour (Damasio et al. 1990). This dysfunction may be key to the pathogenesis of depressive and anxiety comorbidity in UHR subjects. Histopathological studies in MDD have revealed specific morphometric changes in the frontal cortex including the ACC (Rajkowska et al. 1999) that are hypothesized to be due to glial pathology and suggest that such neuroplastic changes could cause a potential dysregulation in the frontolimbic circuit involved in the pathology of depression (Schroeter et al. 2008, 2010). Although largely speculative due to the absence of histopathological evidence from individuals at UHR of psychosis, one possibility for the observed neuroanatomical changes in UHR-co-morbidity subjects would be that they originate as suggested by the histopathologically generated hypothesis of glial pathology in depression, and accompany the morphometric changes typically associated with the UHR. Evidence from a range of methodologies including neuroimaging, lesion analysis and electrophysiological studies of humans and experimental animals all show that reduction in ACC volume and histopathological changes in this area can modulate emotional behaviour and stress responses (Drevets, 2000, 2001). Human lesion studies of the ventral ACC have documented abnormal autonomic responses to emotionally provocative stimuli, decreased emotional experience, and an impact on social behaviour by causing inability to use information regarding the probability of aversive social consequences versus reward in guiding social behaviour (Damasio et al. 1990). Our results tentatively suggest a neural model of depressive and anxiety co-morbidity in UHR subjects in which neuroanatomical changes in the ACC increase the severity of affective symptomatology. Correlation analysis between the positive subscales and the depression and anxiety subscales of the CAARMS yielded no significant associations in the UHR group as a whole, or in the UHR subgroups, providing further support for the idea that neuroanatomical changes in the UHR-co-morbidity group were not simply due to a higher severity of overall psychopathology. Nevertheless, as the first neuroanatomical evidence of this kind, this finding needs to be replicated in larger samples.

To test our second hypothesis, all UHR participants were followed clinically subsequent to scanning to explore the impact of GMV alterations at baseline on symptoms and functioning. Within the UHRco-morbidity group, ACC volume was negatively correlated with the baseline suicidality/self-harm and 

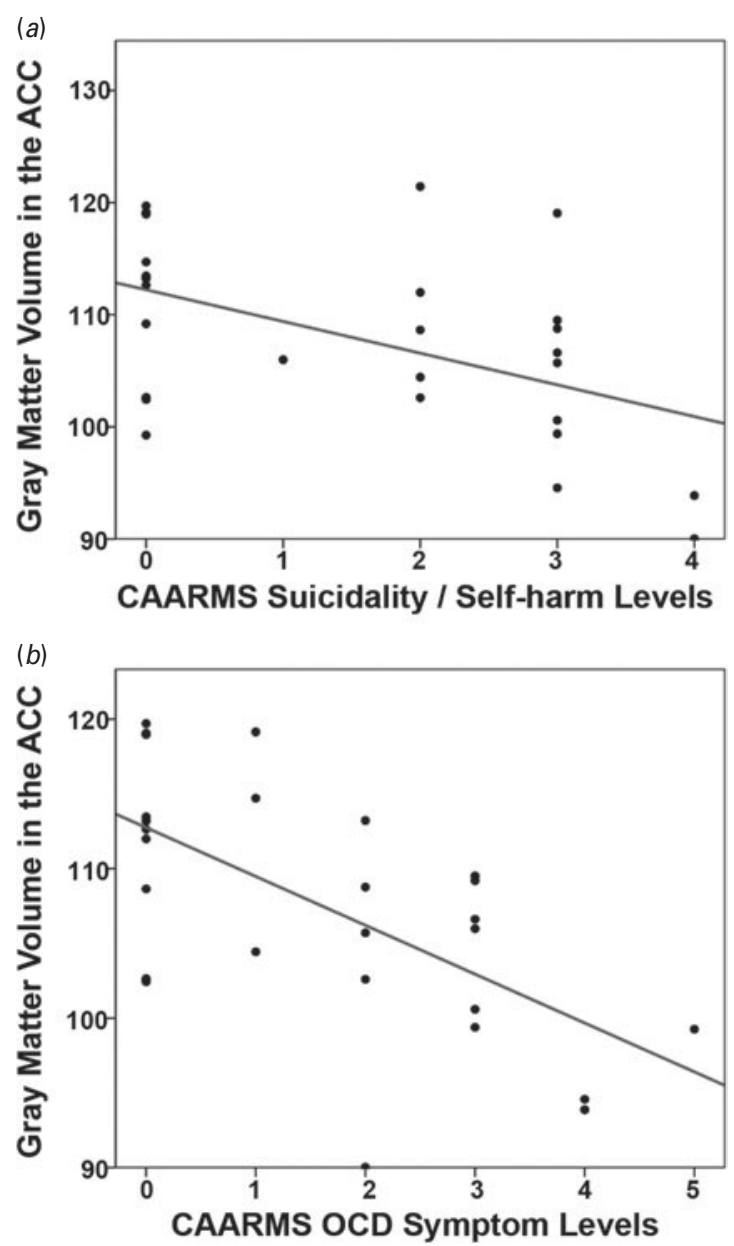

Fig. 3. Correlations between anterior cingulate cortex (ACC) volume and baseline clinical profiles determined using the Comprehensive Assessment of the At-Risk Mental State (CAARMS) in patients at high risk of psychosis with co-morbidity diagnoses. The plots show a significant negative correlation between the grey matter volumes in the ACC and baseline levels of CAARMS suicidality/self-harm symptoms (a) and obsessive-compulsive disorder (OCD) symptoms $(b)$ (higher CAARMS values indicate higher severity of symptoms).

OCD subscales of the CAARMS (showing less volume with increased suicidality and OCD symptoms). The neurobiological association of ACC with suicidal behaviour has been widely supported by neuropathological studies (Hercher et al. 2010; Torres-Platas et al. 2011), studies in subjects at high risk for suicide (Wagner et al. 2012) and studies in patients with previous suicide attempts (Wagner et al. 2011; Pan et al. 2013). These findings were detected in the context of an overall clinical improvement of the UHRco-morbidity subgroup, as indicated by the longitudinal increase in functional levels and by the lack of significant between-group differences in psychopathology at follow-up. These outcomes could be due to spontaneous remission of the co-morbid symptoms, or alternatively to the more intensive treatments offered to these subjects during their clinical management. In fact, the presence of UHR co-morbidity significantly made an impact on the clinical management of UHR subjects during the duration of the study. To the best of our knowledge this is the first report showing that UHR patients with co-morbid depressive or anxiety diagnoses tend to be treated with more medications (antipsychotics and antidepressants) and fewer psychological interventions as stand-alone as compared with the UHR-alone group. Given that antipsychotics and antidepressants in addition to psychological interventions can prevent the onset of psychosis (Fusar-Poli et al. 2007), the longitudinal results of our cohort do not represent the natural history of the UHR state. Furthermore, treatments for the UHR state are commonly non-specific and may target depression and anxiety and not only attenuated symptoms (O'Connor et al. 2007). This model is further supported by recent meta-analytical evidence indicating that ACC structure is particularly sensitive to antipsychotic treatment during the early phases of psychosis (Radua et al. 2012). Additional functional imaging evidence shows that a few weeks of antipsychotic treatment can modulate the ACC response (Lahti et al. 2004; Snitz et al. 2005). Overall, our results support the notion that the UHR state should be considered as a heterogeneous condition in which individuals present with a combination of psychotic and affective (depression, anxiety) symptoms, some of whom, but not all, have a true vulnerability to schizophrenia (Fusar-Poli et al. 2014c). This is discussed in more detail in two recent and comprehensive reviews (Fusar-Poli et al. 2013a, 2014a). The present study provides additional evidence for this idea and demonstrates at a neurobiological level the existence of a depressive-anxiety UHR subtype that is characterized by unique neuroanatomical characteristics along with distinctive psychopathological, therapeutic and prognostic features.

There are some limitations to the present study. First, we lacked an additional group of patients affected with anxiety disorders only. This would have allowed us to better delineate the neuroanatomical circuits associated with depressive versus anxiety disorders. Second, because our UHR-co-morbidity sample was receiving distinctive preventative treatments as compared with the UHR-alone group, the natural history of this subgroup could not be fully assessed. Furthermore, we lacked a follow-up scan to clarify the dynamic longitudinal course of the neuroanatomical alterations observed in our UHR-co-morbidity group. Third, the MDD group was older and the proportion of females was higher compared with the UHR and 
HC groups. Although we modelled these factors as covariates of no interest in GMV analysis, a potential confounding effect cannot be entirely ruled out. Future studies with larger samples are needed to replicate our findings. Information regarding the treatment of UHR subjects during their clinical management at the OASIS clinic (i.e. from baseline to follow-up) was obtained from OASIS patient records.

In summary, our results suggest that depressive and anxiety co-morbidity contributes to specific GMV reductions in UHR subjects, primarily focused in the pregenual ACC. This highlights the neurobiological heterogeneity of UHR, which makes an impact on clinical profiles of UHR individuals both crosssectionally on their clinical management and longitudinal outcomes. Since co-morbid depressive and anxiety diagnoses confer unique psychopathological, neuroanatomical, treatment and prognostic features to UHR individuals, they should be carefully considered in future imaging studies.

\section{Supplementary material}

For supplementary material accompanying this paper visit http://dx.doi.org/10.1017/S0033291714000865

\section{Acknowledgements}

This work was supported by the European network of national schizophrenia networks studying GeneEnvironment Interactions (project EU-GEI; no. HEALTH-F2-2010-241909). We also acknowledge the St Thomas and Guy's Hospital charitable contributions to the OASIS service, which enabled part of the scanning of this sample.

\section{Declaration of Interest}

None.

\section{References}

APA (1994). Diagnostic and Statistical Manual of Mental Disorders, 4th edn. American Psychiatric Association: Washington, DC.

Bora E, Fornito A, Pantelis C, Yucel M (2012). Gray matter abnormalities in major depressive disorder: a meta-analysis of voxel based morphometry studies. Journal of Affective Disorders 138, 9-18.

Borgwardt SJ, McGuire P, Fusar-Poli P, Radue EW, Riecher-Rossler A (2008). Anterior cingulate pathology in the prodromal stage of schizophrenia. NeuroImage 39, 553-554.

Borgwardt SJ, Riecher-Rossler A, Dazzan P, Chitnis X, Aston J, Drewe M, Gschwandtner U, Haller S, Pfluger M,
Rechsteiner E, D'Souza M, Stieglitz RD, Radu EW, McGuire PK (2007). Regional gray matter volume abnormalities in the at risk mental state. Biological Psychiatry 61, 1148-1156.

Broome MR, Woolley JB, Johns LC, Valmaggia LR, Tabraham P, Gafoor R, Bramon E, McGuire PK (2005). Outreach and support in south London (OASIS): implementation of a clinical service for prodromal psychosis and the at risk mental state. European Psychiatry 20, 372-378.

Damasio AR, Tranel D, Damasio H (1990). Individuals with sociopathic behavior caused by frontal damage fail to respond autonomically to social stimuli. Behavioral Brain Research 41, 81-94.

Demjaha A, Valmaggia L, Stahl D, Byrne M, McGuire P (2012). Disorganization/cognitive and negative symptom dimensions in the at-risk mental state predict subsequent transition to psychosis. Schizophrenia Bulletin 38, 351-359.

Drevets WC (2000). Neuroimaging studies of mood disorders. Biological Psychiatry 48, 813-829.

Drevets WC (2001). Neuroimaging and neuropathological studies of depression: implications for the cognitiveemotional features of mood disorders. Current Opinion in Neurobiology 11, 240-249.

First M, Spitzer R, Gibbon M, Williams JBW (1996). Structured Clinical Interview for DSM-IV Axis I Disorders, Clinician Version (SCID-CV). American Psychiatric Press: Washington, DC.

Folstein MF, Folstein SE, McHugh PR (1975). “Mini-mental state". A practical method for grading the cognitive state of patients for the clinician. Journal of Psychiatric Research 12, 189-198.

Fornito A, Yung AR, Wood SJ, Phillips LJ, Nelson B, Cotton S, Velakoulis D, McGorry PD, Pantelis C, Yucel M (2008). Anatomic abnormalities of the anterior cingulate cortex before psychosis onset: an MRI study of ultra-high-risk individuals. Biological Psychiatry 64, $758-765$.

Fusar-Poli P, Borgwardt S, Bechdolf A, Addington J, Riecher-Rossler A, Schultze-Lutter F, Keshavan $\mathbf{M}$, Wood S, Ruhrmann S, Seidman LJ, Valmaggia L, Cannon T, Velthorst E, De Haan L, Cornblatt B, Bonoldi I, Birchwood M, McGlashan T, Carpenter W, McGorry P, Klosterkotter J, McGuire P, Yung A (2013a). The psychosis high-risk state: a comprehensive state-of-the-art review. JAMA Psychiatry 70, 107-120.

Fusar-Poli P, Borgwardt S, Crescini A, Deste G, Kempton MJ, Lawrie S, McGuire P, Sacchetti E (2011). Neuroanatomy of vulnerability to psychosis: a voxel-based meta-analysis. Neuroscience and Biobehavioral Reviews 35, 1175-1185.

Fusar-Poli P, Byrne M, Badger S, Valmaggia LR, McGuire PK (2013b). Outreach and support in South London (OASIS), 2001-2011: ten years of early diagnosis and treatment for young individuals at high clinical risk for psychosis. European Psychiatry 28, 315-326.

Fusar-Poli P, Carpenter WT, Woods SW, McGlashan TH (2014a). Attenuated psychosis syndrome: ready for 
DSM-5.1? Annual Review of Clinical Psychology. Published online 17 January 2014. doi:10.1146/ annurev-clinpsy-032813-153645.

Fusar-Poli P, Nelson B, Valmaggia L, Yung AR, McGuire PK (2014b). Comorbid depressive and anxiety disorders in 509 individuals with an at-risk mental state: impact on psychopathology and transition to psychosis. Schizophrenia Bulletin 40, 120-131.

Fusar-Poli P, Radua J, McGuire P, Borgwardt S (2012). Neuroanatomical maps of psychosis onset: voxel-wise meta-analysis of antipsychotic-naive VBM studies. Schizophrenia Bulletin 38, 1297-1307.

Fusar-Poli P, Valmaggia L, McGuire P (2007). Can antidepressants prevent psychosis? Lancet 370, 1746-1748.

Fusar-Poli P, Yung AR, McGorry P, van Os J (2014c). Lessons learned from the psychosis high-risk state: towards a general staging model of prodromal intervention. Psychological Medicine 44, 17-24.

Hercher C, Canetti L, Turecki G, Mechawar N (2010). Anterior cingulate pyramidal neurons display altered dendritic branching in depressed suicides. Journal of Psychiatric Research 44, 286-293.

Ho BC, Alicata D, Ward J, Moser DJ, O'Leary DS, Arndt S, Andreasen NC (2003). Untreated initial psychosis: relation to cognitive deficits and brain morphology in first-episode schizophrenia. American Journal of Psychiatry 160, 142-148.

Lahti AC, Holcomb HH, Weiler MA, Medoff DR, Frey KN, Hardin M, Tamminga CA (2004). Clozapine but not haloperidol re-establishes normal task-activated rCBF patterns in schizophrenia within the anterior cingulate cortex. Neuropsychopharmacology 29, 171-178.

Mechelli A, Riecher-Rossler A, Meisenzahl EM, Tognin S, Wood SJ, Borgwardt SJ, Koutsouleris N, Yung AR, Stone JM, Phillips LJ, McGorry PD, Valli I, Velakoulis D, Woolley J, Pantelis C, McGuire P (2011). Neuroanatomical abnormalities that predate the onset of psychosis: a multicenter study. Archives of General Psychiatry 68, 489-495.

Meisenzahl EM, Koutsouleris N, Gaser C, Bottlender R, Schmitt GJ, McGuire P, Decker P, Burgermeister B, Born C, Reiser M, Moller HJ (2008). Structural brain alterations in subjects at high-risk of psychosis: a voxel-based morphometric study. Schizophrenia Research 102, 150-162.

Mishara AL, Fusar-Poli P (2013). The phenomenology and neurobiology of delusion formation during psychosis onset: Jaspers, Truman symptoms, and aberrant salience. Schizophrenia Bulletin 39, 278-286.

O'Connor R, Sota M, Cortesi M, Fusar-Poli P (2007). Quetiapine as a first-choice agent in subjects at high-risk to psychosis? Medical Hypotheses 69, 230.

Pan LA, Hassel S, Segreti AM, Nau SA, Brent DA, Phillips ML (2013). Differential patterns of activity and functional connectivity in emotion processing neural circuitry to angry and happy faces in adolescents with and without suicide attempt. Psychological Medicine 43, 2129-2142.

Pantelis C, Velakoulis D, McGorry PD, Wood SJ, Suckling J, Phillips LJ, Yung AR, Bullmore ET, Brewer W, Soulsby B, Desmond P, McGuire PK (2003).
Neuroanatomical abnormalities before and after onset of psychosis: a cross-sectional and longitudinal MRI comparison. Lancet 361, 281-288.

Radua J, Borgwardt S, Crescini A, Mataix-Cols D, Meyer-Lindenberg A, McGuire PK, Fusar-Poli P (2012). Multimodal meta-analysis of structural and functional brain changes in first episode psychosis and the effects of antipsychotic medication. Neuroscience and Biobehavioral Reviews 36, 2325-2333.

Radua J, van den Heuvel OA, Surguladze S, Mataix-Cols D (2010). Meta-analytical comparison of voxel-based morphometry studies in obsessive-compulsive disorder vs other anxiety disorders. Archives of General Psychiatry 67, 701-711.

Rajkowska G, Miguel-Hidalgo JJ, Wei J, Dilley G, Pittman SD, Meltzer HY, Overholser JC, Roth BL, Stockmeier CA (1999). Morphometric evidence for neuronal and glial prefrontal cell pathology in major depression. Biological Psychiatry 45, 1085-1098.

Sacher J, Neumann J, Funfstuck T, Soliman A, Villringer A, Schroeter ML (2012). Mapping the depressed brain: a meta-analysis of structural and functional alterations in major depressive disorder. Journal of Affective Disorders 140, 142-148.

Salokangas RK, Ruhrmann S, von Reventlow HG, Heinimaa M, Svirskis T, From T, Luutonen S, Juckel G, Linszen D, Dingemans P, Birchwood M, Patterson P, Schultze-Lutter F, Klosterkötter J; EPOS Group (2012). Axis I diagnoses and transition to psychosis in clinical high-risk patients EPOS project: prospective follow-up of 245 clinical high-risk outpatients in four countries. Schizophrenia Research 138, 192-197.

Schennach-Wolff R, Jager M, Seemuller F, Obermeier M, Messer T, Laux G, Pfeiffer $H$, Naber D, Schmidt LG, Gaebel W, Huff W, Heuser I, Maier W, Lemke MR, Ruther E, Buchkremer G, Gastpar M, Moller HJ, Riedel M (2009). Defining and predicting functional outcome in schizophrenia and schizophrenia spectrum disorders. Schizophrenia Research 113, 210-217.

Schroeter ML, Abdul-Khaliq H, Krebs M, Diefenbacher A, Blasig IE (2008). Serum markers support disease-specific glial pathology in major depression. Journal of Affective Disorders 111, 271-280.

Schroeter ML, Abdul-Khaliq H, Sacher J, Steiner J, Blasig IE, Mueller K (2010). Mood disorders are glial disorders: evidence from in vivo studies. Cardiovascular Psychiatry and Neurology 2010, 780645.

Snitz BE, MacDonald A 3rd, Cohen JD, Cho RY, Becker T, Carter CS (2005). Lateral and medial hypofrontality in first-episode schizophrenia: functional activity in a medication-naive state and effects of short-term atypical antipsychotic treatment. American Journal of Psychiatry 162, 2322-2329.

Song XW, Dong ZY, Long XY, Li SF, Zuo XN, Zhu CZ, He Y, Yan CG, Zang YF (2011). REST: a toolkit for resting-state functional magnetic resonance imaging data processing. PLoS ONE 6, e25031.

Spellmann I, Riedel $M$, Schennach $R$, Seemuller $F$, Obermeier M, Musil R, Jager M, Schmauss M, Laux G, 
Pfeiffer H, Naber D, Schmidt LG, Gaebel W, Klosterkotter J, Heuser I, Maier W, Lemke MR, Ruther E, Klingberg S, Gastpar M, Moller HJ (2012). One-year functional outcomes of naturalistically treated patients with schizophrenia. Psychiatry Research 198, 378-385.

Thompson A, Nelson B, Yung A (2011). Predictive validity of clinical variables in the "at risk" for psychosis population: international comparison with results from the North American Prodrome Longitudinal Study. Schizophrenia Research 126, 51-57.

Torres-Platas SG, Hercher C, Davoli MA, Maussion G, Labonte B, Turecki G, Mechawar N (2011). Astrocytic hypertrophy in anterior cingulate white matter of depressed suicides. Neuropsychopharmacology 36, 2650-2658.

Tsuang MT, Van Os J, Tandon R, Barch DM, Bustillo J, Gaebel W, Gur RE, Heckers S, Malaspina D, Owen MJ, Schultz S, Carpenter W (2013). Attenuated psychosis syndrome in DSM-5. Schizophrenia Research 150, 31-35.

Valmaggia LR, Stahl D, Yung AR, Nelson B, Fusar-Poli P, McGorry PD, McGuire PK (2013). Negative psychotic symptoms and impaired role functioning predict transition outcomes in the at-risk mental state: a latent class cluster analysis study. Psychological Medicine 43, 2311-2325.

van Os J, Murray RM (2013). Can we identify and treat "schizophrenia light" to prevent true psychotic illness? British Medical Journal 346, f304.

Velthorst E, Nelson B, Wiltink S, de Haan L, Wood SJ, Lin A, Yung AR (2013). Transition to first episode psychosis in ultra high risk populations: does baseline functioning hold the key? Schizophrenia Research 143, 132-137.

Wagner G, Koch K, Schachtzabel C, Schultz CC, Sauer H, Schlosser RG (2011). Structural brain alterations in patients with major depressive disorder and high risk for suicide: evidence for a distinct neurobiological entity? Neuroimage 54, 1607-1614.

Wagner G, Schultz CC, Koch K, Schachtzabel C, Sauer H, Schlosser RG (2012). Prefrontal cortical thickness in depressed patients with high-risk for suicidal behavior. Journal of Psychiatric Research 46, 1449-1455.

Wood SJ, Pantelis C, Velakoulis D, Yucel M, Fornito A, McGorry PD (2008). Progressive changes in the development toward schizophrenia: studies in subjects at increased symptomatic risk. Schizophrenia Bulletin 34, 322-329.

Wulff K, Dijk DJ, Middleton B, Foster RG, Joyce EM (2012). Sleep and circadian rhythm disruption in schizophrenia. British Journal of Psychiatry 200, 308-316.

Yung AR, Phillips LJ, McGorry PD, McFarlane CA, Francey S, Harrigan S, Patton GC, Jackson HJ (1998). Prediction of psychosis. A step towards indicated prevention of schizophrenia. British Journal of Psychiatry. Supplement 172, 14-20.

Yung AR, Woods SW, Ruhrmann S, Addington J, Schultze-Lutter F, Cornblatt BA, Amminger GP, Bechdolf A, Birchwood M, Borgwardt S, Cannon TD, de Haan L, French P, Fusar-Poli P, Keshavan M, Klosterkotter J, Kwon JS, McGorry PD, McGuire P, Mizuno M, Morrison AP, Riecher-Rossler A, Salokangas RK, Seidman LJ, Suzuki M, Valmaggia L, van der Gaag M, Wood SJ, McGlashan TH (2012). Whither the attenuated psychosis syndrome? Schizophrenia Bulletin 38, 1130-1134.

Yung AR, Yuen HP, McGorry PD, Phillips LJ, Kelly D, Dell'Olio M, Francey SM, Cosgrave EM, Killackey E, Stanford C, Godfrey K, Buckby J (2005). Mapping the onset of psychosis: the Comprehensive Assessment of At-Risk Mental States. Australian and New Zealand Journal of Psychiatry 39, 964-971. 